\title{
Effects of Porosity and Thermal Treatment on Hydration of Mushrooms
}

\author{
Ekaraj Paudel $^{1} \cdot$ Remko M. Boom ${ }^{1} \cdot$ Ruud G. M. Van der Sman ${ }^{1}$
}

Received: 2 June 2015 / Accepted: 3 November 2015 / Published online: 18 November 2015

(C) The Author(s) 2015. This article is published with open access at Springerlink.com

\begin{abstract}
In this study, hydration of mushroom as a porous food material has been studied considering their biphasic character. It consists of a solid phase that consists of intertwined hyphae and having cell walls with a swellable polymeric matrix and a pore phase made up by the space in between the hyphae. We have investigated the hydration of mushrooms as a function of initial porosity and thermal treatment. Variation in porosity is induced by the natural variation in the growth of mushroom. Porosity is measured by the weight gain during vacuum infiltration of mushroom caps with the mushroom isotonic solution. The hydration of the heat-treated mushroom shows a linear increase with the porosity of mushroom. Storage of mushroom increases the porosity with the number of storage days, which has also been confirmed with X-ray tomography(XRT) measurements. Finally, we show that the hydration of thermal-treated mushroom has two independent contributions, from porosity and temperature of the heat treatment. Current theories deal only with the latter contribution to the hydration of foods and need to be extended for accounting porosity of the food.
\end{abstract}

Keywords Mushroom processing $\cdot$ Porosity $\cdot$ Degree of hydration $\cdot$ Water-holding capacity

\section{Introduction}

In the last recent years, we have been researching a mechanistic explanation for the water-holding capacity (WHC) of food

Ekaraj Paudel

ekaraj.paudel@wur.nl; pyogen2002@gmail.com

1 Laboratory of Food Process Engineering, Wageningen University, P.O. Box 17, 6700 AA Wageningen, The Netherlands materials using the Flory-Rehner (FR) theory. We have applied that theory to meat (van der Sman 2012), freeze-dried vegetables (van der Sman et al. 2013), and mushrooms (Paudel, Boom, and van der Sman 2015). Theoretically, the FR theory only applies to gel-like materials. Some foods like freeze-dried vegetables clearly have both a gel phase and a pore phase. The gel phase consists mainly of cell wall material which swells upon hydration. In the pore phase, the water is held by capillary forces. In our previous research (van der Sman et al. 2013), we have not been able to separate these two different contributions to WHC because of the leaching of solutes from the gel phase to the pore phase.

In this paper, we analyze the effect of porosity on the WHC of mushrooms, which is obtained by separating its contribution from swelling of the gel phase. Fresh mushrooms have clearly a distinct pore space, filled with air. The gel phase consists of intertwined hyphae. Its dry matter consists mainly of cell wall material and carbohydrates in the vacuole. In our previous paper (Paudel et al. 2015), we have investigated the WHC of vacuum-infiltrated mushrooms via centrifugation. Via vacuum infiltration, we have filled the pore space with an isotonic liquid having the same composition as the intracellular fluid. At sufficient high centrifugation speeds, the pore phase has collapsed. Furthermore, the swelling of the gel phase due to difference in osmotic pressure between gel and pore phase is prevented via vacuum impregnation of the pore phase with an isotonic fluid. By these two measures, we have been able to determine the WHC of only the gel phase.

Other systems having both a gel phase and a pore phase are freeze-dried fruits and vegetables and coarse gels. Properties of the freeze-dried systems are already discussed (van der Sman et al. 2013). Coarse, particulate gels can be viewed as a network of aggregated microgel particles with a liquid-filled pore phase (Leksrisompong et al. 2012; Nicolai and Durand 2013). This is in contrast to fine-stranded gels, where the biopolymers form a space-filling network. The water holding of these fine-stranded gels can be fully explained by the Flory- 
Rehner theory and is linear with their gel strength (van der Sman 2015). Water holding of coarse gels is little explained by gel strength, but rather by the size of the pore (Urbonaite et al. 2015). Theoretical investigation of the contribution of the pore space to the water holding of particulate gels is hampered by the fact that pore size is often in the submicron range, where both the van der Waals forces and the capillary forces contribute to the water binding in the pore space (Shahraeeni and Or 2010; Tuller, Dani, and Dudley 1999).

In mushrooms, the pore size measures several micrometers, and the van der Waals forces can safely be assumed negligible compared to the capillary forces. Hence, we view mushroom as a good model system for a detailed investigation of the contributions of the gel phase and the pore phase to the water-holding capacity. The pore space is well open to the environment and can easily be filled with liquid via vacuum impregnation. The natural variability in porosity of mushrooms and its maturation render a large variation in porosity, namely in the range of 0.25 to 0.50 , which gives us a means to vary the contribution of the pore phase. On the other hand, temperature treatment gives us a means to alter the contribution of gel phase on the water-holding capacity. At high temperatures, proteins present in the cell wall materials denature, which lowers the water binding of the gel phase. During storage, mushrooms are still physiologically active and mature. Prolonged maturation has shown an increase in their hydration after a thermal treatment (Beelman, Kuhn, and McArdle 1973; Beelman and McArdle 1975; Zivanovic, Busher, and Kim 2000). While the reason for such an increase is attributed to the proteolytic changes in protein (Beelman, Kuhn, and McArdle 1973; Beelman and McArdle 1975), this paper gives the insight that maturation influences the hydration properties via porosity changes.

\section{Methods and Materials}

\section{General Methodology}

An important measure for the contribution of the pore space to the WHC is its volume fraction, i.e., the porosity. The global value of porosity has been measured via weight gain following vacuum impregnation with an isotonic fluid. As reported in our previous paper (Paudel et al. 2015), fluid with the composition equal to cellular fluid does not lead to swelling of the gel phase. Hence, the isotonic fluid just fills the pore while displacing air during the vacuum impregnation. Next to the global volume fraction of the pore phase, we have investigated on its local variation via X-ray tomography (XRT). Mushrooms with different global porosity values were obtained from their biological variation and after their maturation. To enhance their porosity via maturation, the mushrooms are stored at a temperature of $4{ }^{\circ} \mathrm{C}$ and $95 \% \mathrm{RH}$ prior to the vacuum impregnation. The contribution of gel phase in WHC changes with the thermal treatment as a result of protein denaturation. This change in the contribution with the temperature is studied by giving a thermal treatment to the mushrooms. Porosity is determined via vacuum infiltration for every mushroom. Water loss is measured at different temperatures of thermal treatment. Collapse phenomenon of the pore phase as a result of thermal treatment is studied by giving the thermal treatment to mushrooms in which pores were previously partially filled with the isotonic solution. We have used white button mushroom (Agaricus bisporus) with a cap diameter in the range of 4 to $5 \mathrm{~cm}$ for all our experiments. The mushrooms were received within $2 \mathrm{~h}$ of their harvest and stored at temperature of $4{ }^{\circ} \mathrm{C}$ and $95 \% \mathrm{RH}$ until they were used for experiments.

\section{Formulation of Isotonic Solution}

The mushroom isotonic solution contained $2.2 \%$ mannitol, $0.2 \%$ trehalose, and $0.8 \%$ potassium phosphate dissolved in deionized water and was adjusted to a native $\mathrm{pH}$ value 6.3 of fresh mushrooms, as suggested in our previous paper (Paudel et al. 2015). The solution was used both for measuring porosity of fresh mushroom and as a heat transfer medium providing the thermal treatment to the mushroom samples.

\section{Estimation of Porosity of Mushroom with Isotonic Solution}

Porosity of mushroom is computed by the weight gain of mushroom after its vacuum impregnation with the isotonic solution. Caps of mushrooms are submerged in the isotonic solution and then subjected to a set vacuum of 20 mbar at room temperature for $10 \mathrm{~min}$. The vacuum is then released, and the caps were kept in the isotonic solution for another $5 \mathrm{~min}$ to allow full impregnation of the solution into the pores. The porosity, volume fraction of air in mushroom, was calculated following a simple mass balance as is described in Eq. 1:

$$
\in=\frac{\left(W_{\mathrm{H}}-W_{\mathrm{F}}\right) / \rho_{\text {is }}}{\left(W_{\mathrm{H}}-W_{\mathrm{F}}\right) / \rho_{\text {is }}+{ }^{W_{F}} / \rho_{\mathrm{th}}}
$$

Here, $W_{\mathrm{F}}$ and $\mathrm{W}_{\mathrm{H}}$ are the weight of mushroom before and after the vacuum impregnation, $\rho_{\text {is }}$ is the density of isotonic solution, and $\rho_{\text {th }}$ is the theoretical density of the gel phase of mushroom calculated from its composition (Paudel et al. 2015): $92.1 \%$ water $\left(\rho_{\mathrm{w}}=1000\right), 2.3 \%$ sugar $\left(\rho_{\mathrm{s}}=1550\right)$, $2.6 \%$ polysaccharides $\left(\rho_{\mathrm{c}}=1550\right), 2.1 \%$ protein $\left(\rho_{\mathrm{p}}=\right.$ 
$1330)$, and $0.8 \%$ minerals $\left(\rho_{\mathrm{m}}=2440\right)$. The theoretical density was then calculated from the individual densities following the relation (van der Sman 2008):

$$
\frac{1}{\rho_{\mathrm{th}}}=\sum_{i} \frac{Y_{i}}{\rho_{i}}
$$

\section{WHC Measurements}

The vacuum-impregnated mushrooms are stored at $4{ }^{\circ} \mathrm{C}$ for $24 \mathrm{~h}$ and are subjected to a thermal treatment with a temperature-controlled bath holding the isotonic solution (1:5 weight ratio of hydrated mushroom to the isotonic solution). After the thermal treatment, mushrooms are taken out of the bath and cooled in a bath of the isotonic solution held at room temperature. The cooled mushrooms are allowed to drain surface attached water, and their surface are dried with a tissue. Subsequently, their weight was measured. Waterholding capacity is expressed in terms of relative degree of hydration $\left(Y_{\mathrm{H}}\right)$ as the ratio of the weight of the heat-treated sample $\left(W_{\mathrm{T}}\right)$ compared to their fresh weight $\left(W_{\mathrm{F}}\right)$ before vacuum impregnation.

$Y_{\mathrm{H}}=\frac{W_{\mathrm{T}}}{W_{\mathrm{F}}}$

For storage study, mushrooms were stored without vacuum impregnation for three consecutive days. The stored mushrooms were given the vacuum impregnation and the thermal treatment without any storage in between.

\section{Different Degrees of Air Trapping}

The pore phase in mushroom is filled with the isotonic solution to various degrees, and the effect on the hydration behavior after thermal treatment is studied. As filling of pores depends on the vacuum applied, various suction pressures, 1000 , $500,300,100$, and $20 \mathrm{mbar}$, were applied to have partial infiltration of mushrooms with the isotonic solution. The fraction of the pores filled is obtained from the weight gain during the infiltration. Afterwards, mushrooms are provided a thermal treatment at $90{ }^{\circ} \mathrm{C}$ for $15 \mathrm{~min}$ and WHC was measured as described above.

\section{XRT Imaging}

$\mathrm{XRT}$ is used as a measure for the local porosity change during storage. Two mushrooms are analyzed with XRT for three consecutive days. During storage, the mushrooms are kept in a refrigerator at $4{ }^{\circ} \mathrm{C}$. For imaging, the mushroom are taken out of the refrigerator and are put back after imaging for half an hour. The samples are imaged using the X-ray microfocus CT system (General Electrics, Phoenix). A power setting of
$70 \mathrm{kV}$ voltage and $350 \mu \mathrm{A}$ currents are used. The transmission of the conical X-ray beam through the sample is recorded by a CCD camera. The sample is rotated with a step size of $0.18^{\circ}$, and the beam of X-ray is recorded by the camera for an acquisition time of $250 \mathrm{~ms}$. The process is repeated 2000 times to get a full $360^{\circ}$ rotation of the sample. The total readout time is $33 \mathrm{~min}$. The $3 \mathrm{D}$ density map is obtained after tomographical reconstruction of images with the software of the instrument. Reconstructed images are analyzed for their (local) porosity values.

The reconstructed XRT image is analyzed in Avizo 8.1 image analysis software package. Among the series of reconstructed images, an image at the center of the mushroom is taken for the measurement of their greyscale value (Fig. 1). A line probe is selected for calculating the greyscale value along the axis of the probe. The greyscale value in the probe is analyzed in a sequence of cylindrical disks with $2 \mathrm{~mm}$ in radius and $50 \mu \mathrm{m}$ in height along with the probe from the top of the mushroom to its bottom as shown in the diagram. A greyscale value of 320 is assigned to the solid phase of the mushroom and 0 for the pore phase. The value of the solid phase is determined via preparing a paste, where all air is removed via vacuum treatment.

\section{Results and Discussion}

\section{Porosity of Fresh Mushroom and the Degree of Hydration After Thermal Treatment}

A large number of batches of mushrooms are examined to find a relation between the porosity and the water-holding capacity after the thermal treatment. A higher degree of hydration after the thermal treatment is found for mushrooms with higher porosity, as indicated in Fig. 2. It shows the measurements for individual mushrooms in three different batches (batch I:

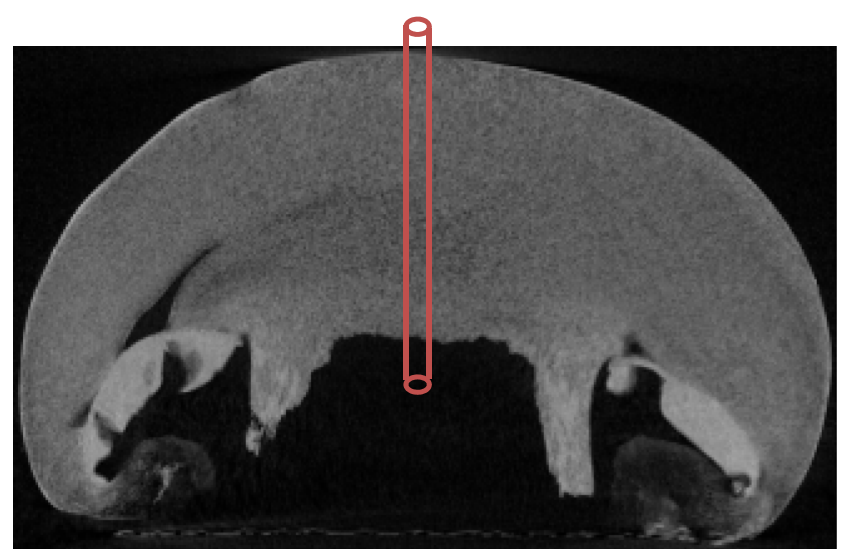

Fig. 1 The schematic diagram of sampling of mushroom in Avizosoftware 


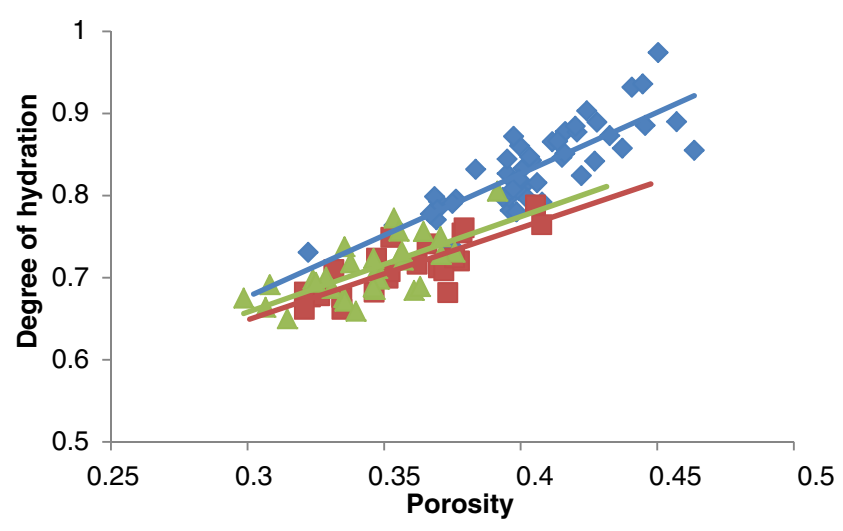

Fig. 2 The relationship between porosity of mushrooms and degree of hydration after thermal treatment (the thermal treatment was provided at $90{ }^{\circ} \mathrm{C}$ for $15 \mathrm{~min}$ ) for three batches of mushroom

blue, batch II: Green and batch VIII: red; Fig. 3). Via linear regression, we obtain correlation coefficients $R^{2}$ value in the range of $0.5-0.7$ for mushroom of various batches. Figure 3 shows the variation of porosity between various batches. The coefficient of linear regression values between the batches were not significantly different to each other. Ignoring the differences between batches and assuming the differences are due only to variation in the initial porosity improved the correlation coefficient value to 0.90 .

While porosities of individual mushroom seem to play an important role in determining their degree of hydration after the thermal treatment, there is a considerable variation in porosities of mushrooms within a batch as shown in Fig. 2 and between the batches as shown in Fig. 3. Though mushrooms among various batches used in the current study were on the same stage of physiological growth, the porosity value of mushroom ranged from 0.25 to 0.5 .

\section{Change in Mushroom Hydration with Temperature}

The change in the degree of hydration of mushroom as a function of porosity for various temperatures is shown in Fig. 4. We observe that the degree of hydration of heat-treated mushroom increases with porosity for all temperatures. At equal porosity, the degree of hydration decreases with the treatment temperature, as reported in several previous studies (Baldwin et al. 1986; Paudel et al. 2015; Schijvens et al. 1996; van der Sman et al. 2013; Zivanovic, Buescher, and Kim 2003).

We have performed linear regression to the data in Fig. 4 and have determined the slope and intercept of the fitted lines, including the error in their estimates. There are no significant differences in the slopes of the regression lines for different temperatures. However, the intercept values can be categorized into three groups. The values are not significantly different to each other within the group, but are significantly different among the groups: (a) 30 and $40{ }^{\circ} \mathrm{C}$; (b) $50{ }^{\circ} \mathrm{C}$; (c) $60,70,80$, and $90{ }^{\circ} \mathrm{C}$. The intercept of the line does decrease with temperature.

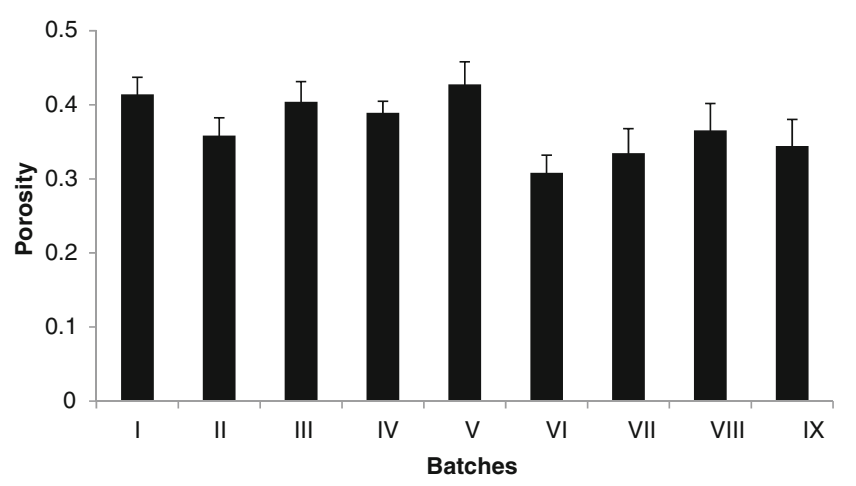

Fig. 3 The variation of porosities between various batches examined. The porosity values are obtained by the weight gain during the vacuum impregnation

The data from Fig. 4 suggest that the degree of hydration of mushroom has two major, independent contributions: the temperature of the thermal treatment and then the initial porosity of the mushrooms. The temperature dependency we attribute to the gel phase of the mushroom, which is shown to depend on temperature following a sigmoid relation (Paudel et al. 2015). The largest change in the degree of hydration happens in the temperature range of $50-60{ }^{\circ} \mathrm{C}$. Figure 4 also shows the largest change in hydration in this temperature range. We assume that the hydration of whole mushroom follows the same sigmoid relation as the gel phase and that it depends linearly on the porosity. This can be expressed as follows:

$Y(T, \in)=\frac{\rho_{\text {is }}}{\rho_{\text {th }}}\left(\frac{\epsilon}{1-\epsilon}\right)+\left(Y_{30}^{0}-\frac{Y_{30}^{0}-Y_{90}^{0}}{1+\exp \left(b\left(T_{0}-T\right)\right)}\right)$

where $Y_{30}^{0}$ and $Y_{90}^{0}$ are the degree of hydrations of gel phase of mushroom at temperature 30 and $90^{\circ} \mathrm{C}$, respectively. $T_{0}$ is the midpoint temperature of the sigmoid curve, and $b$ is the constant whose values are obtained from the curve fitting of the degree of hydration against the temperature for various mushroom porosities. The first and second terms in Eq. (4) represent the weight ratio of fluid present in the capillaries and the gel phase compared to the weight before vacuum impregnation, respectively. The first term is obtained from the rearrangement of $\mathrm{Eq}$ (1), the second term is adopted following our previous study (Paudel et al. 2015).

We have fitted Eq. (4) to the complete dataset as shown in Fig. 4 via non-linear regression, which is shown in Fig. 5. We have obtained the following values for the model parameters: $Y_{30}^{0}=1.01 \pm 0.01, Y_{90}^{0}=0.17 \pm 0.01, b=0.30 \pm 0.02, T_{0}=$ $54.15 \pm 0.37{ }^{\circ} \mathrm{C}$. The values of the constants $b$ and $T_{0}$ agree with the values obtained in our previous paper (Paudel et al. 2015). The goodness of fit is computed via the RMSE, having a value of 0.0392 . Hence, the data analysis confirms that the gel phase and the pore phase contribute independently to the hydration of mushrooms, with only the contribution of the gel phase being temperature dependent. 
Fig. 4 The degree of hydration of mushroom after the thermal treatment as a function of temperature and porosity

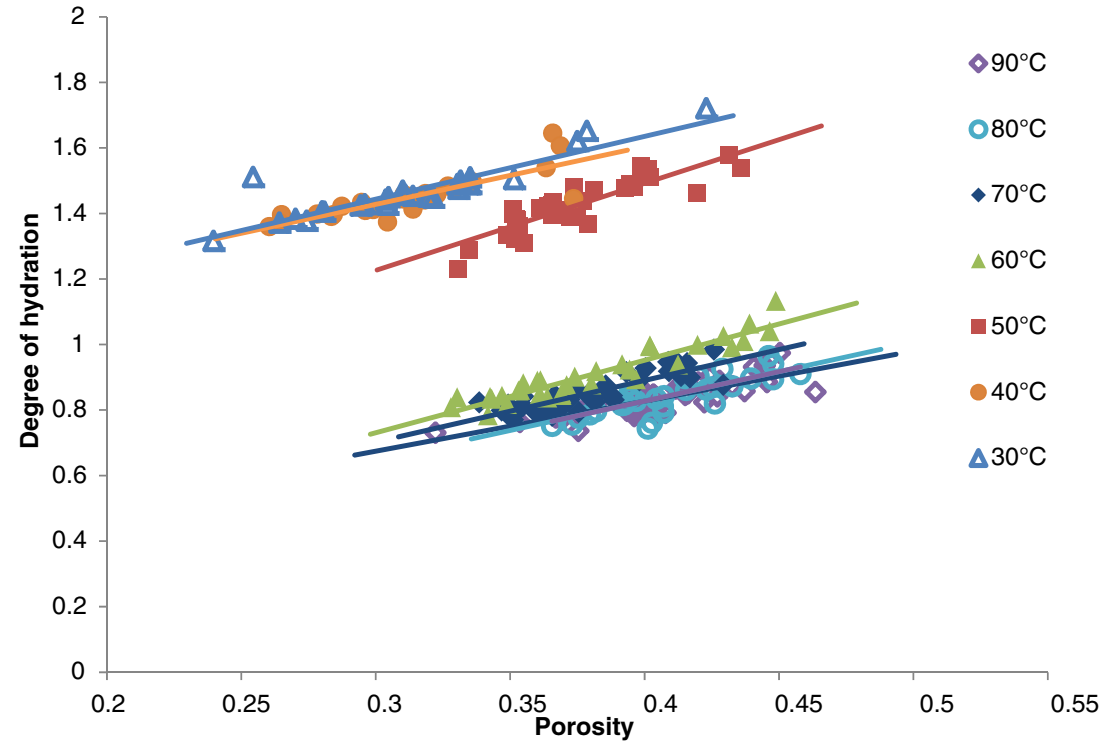

\section{Change in Mushroom Porosity and Degree of Hydration upon Storage}

Figure 6 (left: batch IX and right: batch V, Fig. 3) shows the change in porosity in mushroom and the degree of hydration as a function of storage days for two batches of mushroom. Despite the large natural variation in porosity of individual mushrooms, the values for both the porosity and degree of hydration increased with the storage days. An analysis of covariance (ANCOVA) test showed that the values of the degree of hydration for those lots increased significantly $(p<0.05)$ until the second day of storage. For longer storage, there are no significant changes. We have also performed linear regression on the data (hydration versus porosity). Due to the natural variation, there are no significant differences in the slope of the regression lines. As expressed by Eq. (4), we have not expected any difference in the slope. We assume that storage only influences the intercept of the relation of hydration vs. porosity. With the assumption of a constant slope, we have performed the linear regression again to obtain more accurate estimations of the intercepts.

The intercept in two batches increased significantly $(p<0.05)$ until day 1 and day 2 of storage, respectively. The change in the intercept with storage indicates that storage not

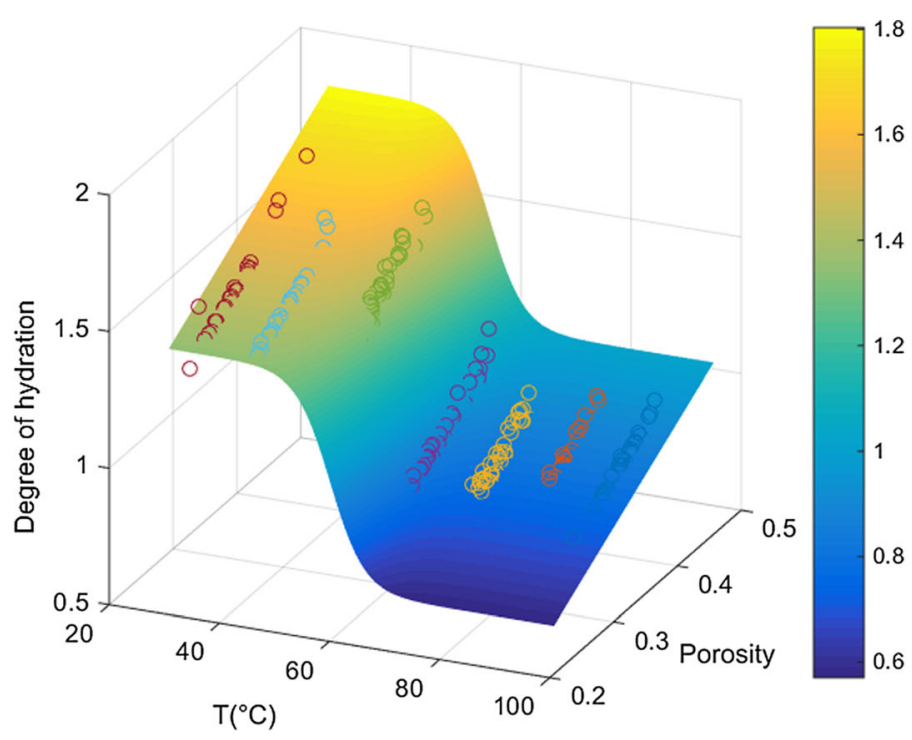

Fig. 5 Left: prediction of the degree of hydration of heat-treated mushroom at various temperatures. The lower and upper boundaries of the sigmoid curves show the prediction at 0.25 and 0.5 , respectively. The

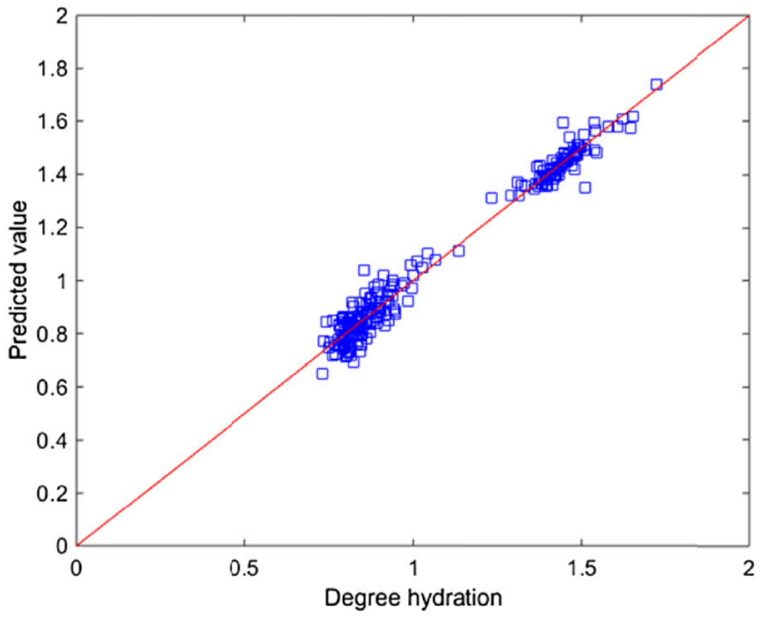

open circles show the experimental data point used for the curve. Right: a plot of real degree of hydrations values against the predicted values 

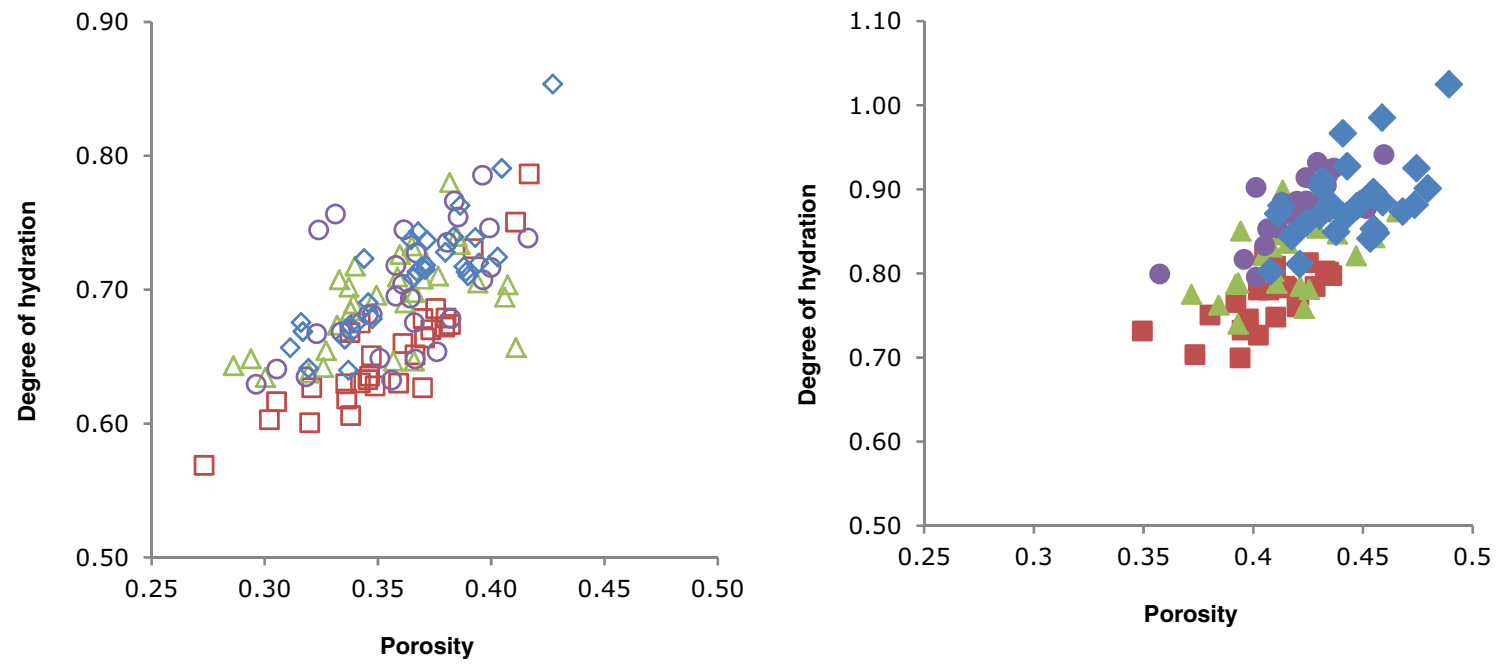

Fig. 6 The degree of hydration of heat-treated mushroom $\left(90^{\circ} \mathrm{C} / 15 \mathrm{~min}\right.$ ) plotted against their porosity for day zero (red square), one (green triangle), two (purple circle), and three (blue diamond). The open and closed symbols represent two different batches

only induces change in porosity but also induces other physiological changes in the gel phase of mushroom altering the hydration of stored mushroom. It is known in literature that the storage of mushroom brings about several biochemical changes in the gel phase of mushroom, including a decreased level of mannitol, total and soluble protein content, and glucan level and an increase in structural components chitin and cell wall proteins (Ajlouni et al. 1995; Hammond and Hammond 1979; Hammond and Nichols 1975; Zivanovic et al. 2000). In the fungal cell wall, chitin provides mechanical strength to the cell wall (Bowman and Free 2006; Zivanovic et al. 2000). With the storage, both the space within the hyphae and their mechanical strength increases because of increased chitin level. This increased space between the hyphae leads to an increased capacity of mushroom to hold more water. The higher mechanical strength keeps mushroom relatively firmer when it is subjected under the thermal treatment. The hydration of the gel phase might have increased due to increase in chitin and cell wall protein level with storage.

\section{Change in Porosity During Storage as Measured by XRT}

The change in the local porosity of mushrooms as measured by X-ray tomography is shown in Fig. 7. We observe that both of the scanned mushrooms show a decrease in the greyscale value with prolonged storage, indicating an increase of porosity in the stored mushroom.

The change in the solid fraction expressed as the greyscale value is shown in Fig. 8. The greyscale value of mushroom tissue is lower than the value measured independently for evacuated mushroom paste, which shows a greyscale value of 320. There are various regions with localized density in mushroom (Schijvens et al. 1996), as is also indicated in Fig. 7. We have quantified this variation for the central axis of the mushroom. Results are shown in Fig. 8. The result shows a drop in greyscale value in both the mushrooms with storage days indicating an increased porosity in mushroom with storage days. The figure shows a higher density (lower porosity) at the top of the mushroom cap with storage. The cap
Fig. 7 The change in mushroom with the storage days as shown by XRT imaging. Lowering white intensity with the storage days indicates a lower density of mushroom as a result of increased pore spaces in the tissue
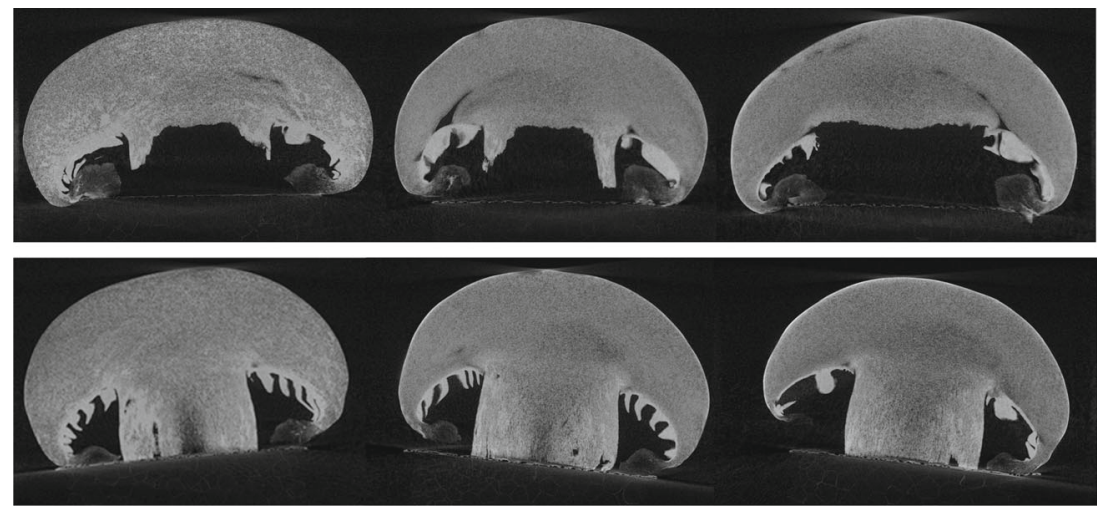

Day 0

Day 1

Day 2 

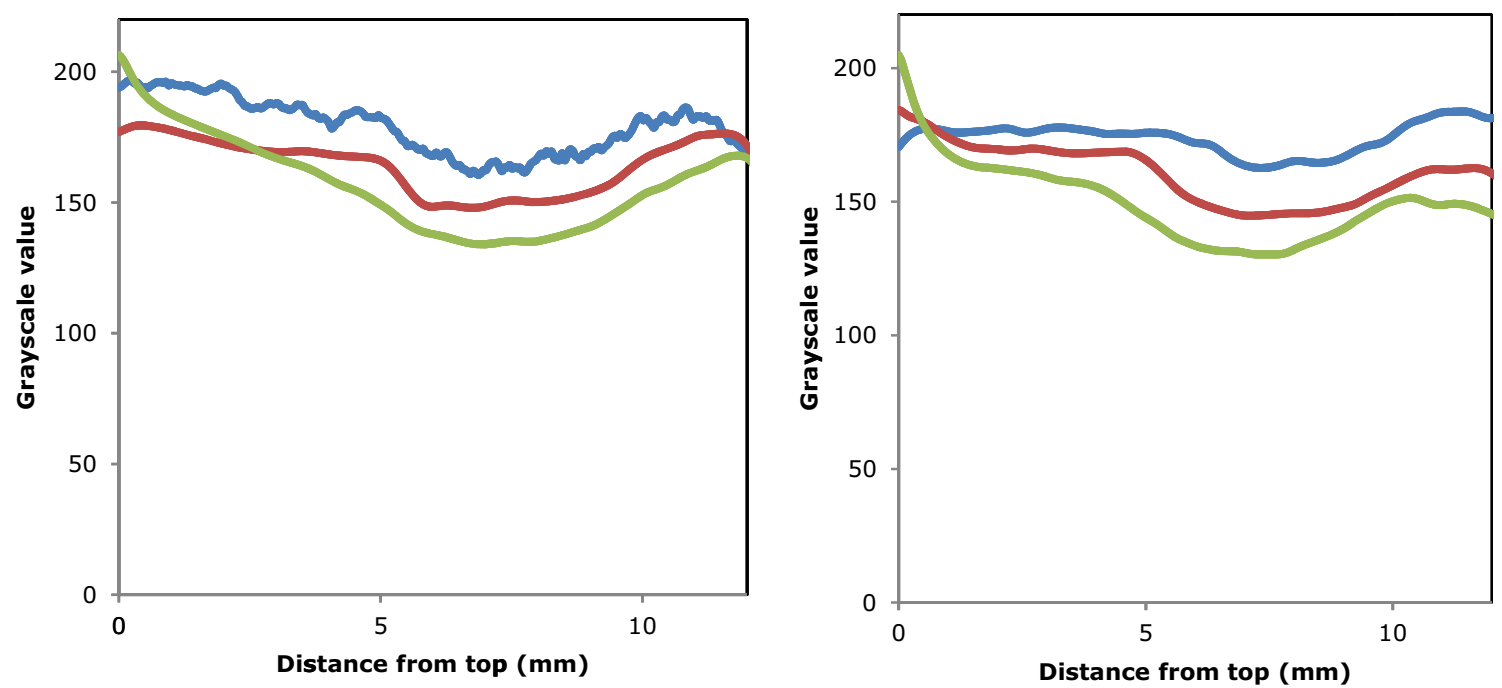

Fig. 8 The greyscale values of the two mushrooms during stored after zero (blue), one (red), and two (green) days of storage

surface is reported to be more porous, having larger openings (Zivanovic et al. 2000) with a lower density than the rest of the tissue (Schijvens et al. 1996). The increased density at the surface in the stored mushroom might be due to surface drying of mushroom with storage days.

\section{Degree of Hydration After Thermal Treatment in Relation to the Filling of Mushroom Pores}

A plot of the fraction of pores filled against the degree of hydration after the thermal treatment of the vacuum-infiltrated mushrooms is shown in Fig. 9. The degree of hydration increases linearly with the fractions of the pores filled as shown in the figure. A similar observation is reported in several previous studies (Anantheswaran et al. 1986; Beelman and McArdle 1975; Lin, Chen, and Lin 2001; McArdle, Kuhn, and Beelman 1974) which report a beneficial effect of infiltration of mushroom to the hydration after the thermal treatment.

We argue that the decreased hydration in mushrooms with partially filled capillaries is because of pore collapse. Pore collapse occurs if the capillary forces in the partially filled capillaries exceeds the critical buckling stress of the wall matrix
Fig. 9 The relation between the fraction of pores filled with the degree of hydration of heattreated mushroom. The heat treatment is carried out at $90{ }^{\circ} \mathrm{C}$ for $15 \mathrm{~min}$

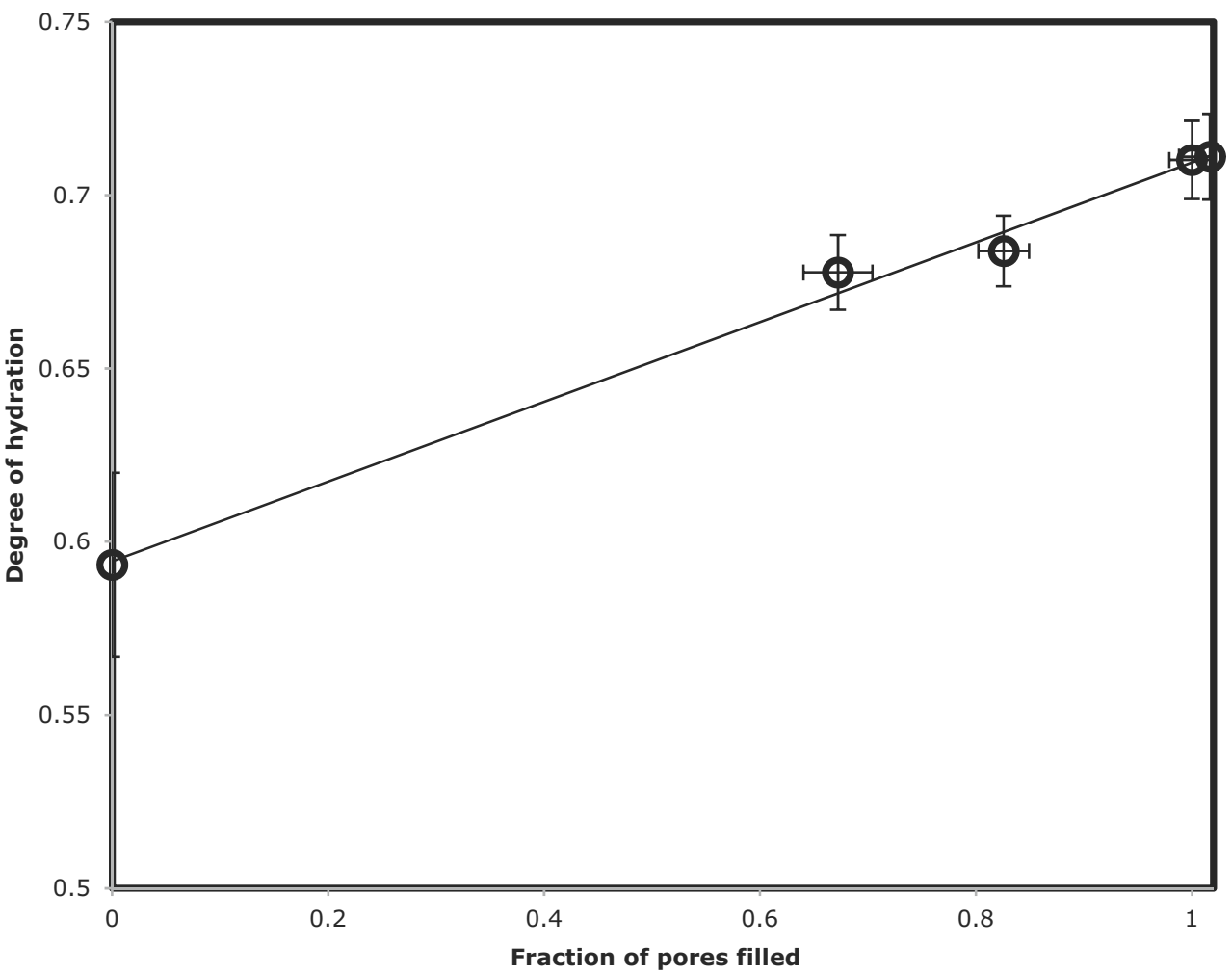


Fig. 10 The conceptual diagram to describe water loss in mushroom with partially filled capillaries. The small arrows show the direction of collapse of hyphae, the big arrows show the pressure exerted by water against collapse. Left: before thermal treatment. Middle: partial collapse of hyphae during the thermal treatment. Right: after the thermal treatment
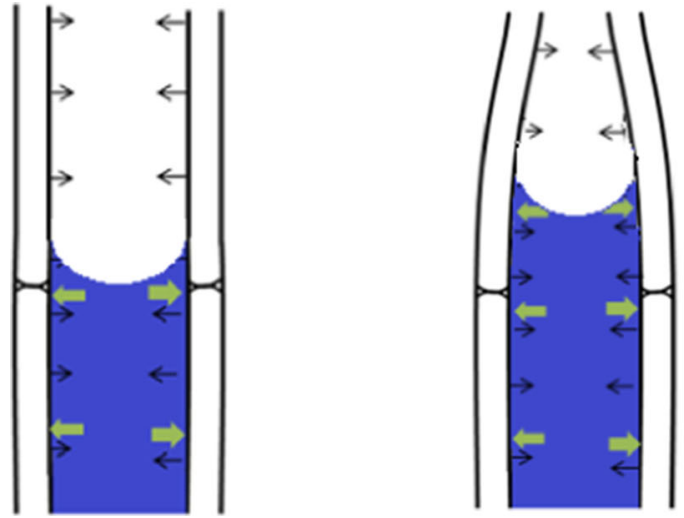

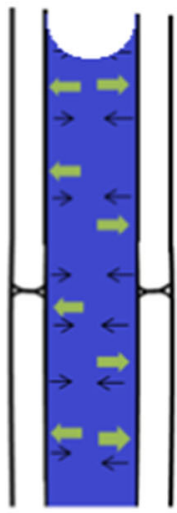

(Heil et al. 2008). A schematic diagram of the phenomenon is shown in Fig. 10. Due to the limited amount of water, the capillary forces will be larger than in fully filled capillaries. The thermal treatment makes the wall less stiff because of protein aggregation which is evident by an increase in the crosslink density of gel phase after the treatment (Paudel et al. 2015). The loss of stiffness makes the wall prone to collapse (Fig. 10, middle) because of the capillary force. The thermal treatment also causes the gel phase to release water. After thermal treatment of the partially filled mushrooms, no porosity is present in the system which is checked with XRT. The capillaries will be fully filled (Fig. 10, right), but the limited amount of water induces a higher curvature of the water meniscus at the mushroom/air interface. This higher curvature induces higher capillary forces, which makes the mushroom shrink more. The capillary force is balanced by the elastic forces due to entanglements of the hyphae, which brings a new equilibrium in the shrinkage. Meat is a food material, whose WHC is investigated quite extensively. Water population in meat is grossly divided into the intramyofibrillar water, which is water present in the muscle fiber by hydrogen bonding and physical entrapment, and capillary water or free water which is water present in cylindrical extracellular cylindrical capillaries in the sarcoplasmic area (Huff-Lonergan and Lonergan 2005; van der Sman 2012). In meat, the thermal processing causes a decrease in intramyofibrillar water population and an increase in water population in the capillary phase (Pearce et al. 2011). The thermal treatment contracts collagen, making this extracellular water to expel. Capillary phase water population in mushroom seems to play even a bigger role in mushroom hydration because of its higher porosity; pore phase holds around $40 \%$ of water in a vacuum-infiltrated fresh mushroom. Unlike meat, the capillary in mushroom is not an anistropic narrow cylindrical passage, but it is a larger space between the hyphae which are randomly intertwined to each other. Upon thermal treatment, hyphae having reduced mechanical strength reach closer to each other that subsequently results in water removal. This phenomenon is in contrast to water loss behavior shown by other vegetables having lower capillary space such as carrot which can still retain relatively more water than mushroom after its thermal treatment (van der Sman et al. 2013). Next to the contribution of capillary phase, chemical makeup of gel phase might also determine hydration behavior as every constituent contribute differently on it. Further research in this area showed that the cell membrane integrity plays a vital role in determining the hydration behavior of mushroom, which will be discussed in our forthcoming paper.

\section{Conclusion}

A description of the hydration behavior of a gel matrix in many food materials is possible with Flory-Rehner's theory as is shown by several previous studies. Many food materials such as mushrooms are more complex than just a system consisting of only a gel matrix. Mushroom serves as a good model system to understand the hydration behavior of a system that consists of a gel phase and a pore phase. The current study shows that porosity and temperature contribute independently to the hydration of heat-treated mushroom. For a constant porosity value, an increase in temperature from 30 to $90{ }^{\circ} \mathrm{C}$ lowers the hydration by half, while for the heattreated mushroom, an increase in initial porosity of mushroom from 0.3 to 0.4 increases their hydration by $20 \%$.

The contribution of capillary water in food material might be different among many foods because of various porosity characteristics and wetting properties of capillary wall. Similarly, WHC of the gel phase of various food materials might change differently with temperature because of variation in the constituents that make them. Knowledge of the composition of the cellular constituents and capillary phase contribution is needed to understand the WHC character of many food materials, including meat and coarse protein gels.

Acknowledgments We are grateful to $\mathrm{C} 4 \mathrm{C}$ holdings $\mathrm{B} . \mathrm{V}$., The Netherlands, for the financial support. We are also thankful to Erik Esveld, Food and Biobased Research (FBR) group of Wageningen University, for his help with the XRT analysis. 
Open Access This article is distributed under the terms of the Creative Commons Attribution 4.0 International License (http:// creativecommons.org/licenses/by/4.0/), which permits unrestricted use, distribution, and reproduction in any medium, provided you give appropriate credit to the original author(s) and the source, provide a link to the Creative Commons license, and indicate if changes were made.

\section{References}

Ajlouni, S. O., Beelman, R. B., Thompson, D. B., Mau, J.-L., \& George, C. (1995). Changes in soluble sugars in various tissues of cultivated mushrooms, Agaricus bisporus, during postharvest storage. Developments in Food Science, 37, 1865-1880.

Anantheswaran, R. C., Sastry, S. K., Beelman, R. B., Okereke, A., \& Konanayakam, M. (1986). Effect of processing on yield, color and texture of canned mushrooms. Journal of Food Science, 51(5), $1197-1200$

Baldwin, D. R., Anantheswaran, R. C., Sastry, S. K., \& Beelman, R. B. (1986). Effect of microwave blanching on the yield and quality of canned mushrooms. Journal of Food Science, 51(4), 965-966.

Beelman, R. B., \& McArdle, F. J. (1975). Influence of post harvest storage temperatures and soaking on yield and quality of canned mushrooms. Journal of Food Science, 40(4), 669-671.

Beelman, R. B., Kuhn, G. D., \& McArdle, J. (1973). Influence of postharvest storage and soaking treatment on the yield and quality of canned mushrooms. Journal of Food Science, 38(6), 951-953.

Bowman, S. M., \& Free, S. J. (2006). The structure and synthesis of the fungal cell wall. BioEssays: News and Reviews in Molecular, Cellular and Developmental Biology, 28(8), 799-808.

Van der Sman, R. G. M. (2012). Thermodynamics of meat proteins. Food Hydrocolloids, 27(2), 529-535.

Hammond, B. W., \& Hammond, J. B. W. (1979). Changes in composition of harvested mushrooms (Agaricus bisporus). Phytochemistry, 18(3), 415-418.

Hammond, J. B. W., \& Nichols, R. (1975). Changes in respiration and soluble carbohydrates during the post-harvest storage of mushrooms (Agaricus bisporus). Journal of the Science of Food and Agriculture, 26(6), 835-842.

Heil, M., Hazel, A. L., \& Smith, J. A. (2008). The mechanics of airway closure. Respiratory Physiology \& Neurobiology, 163, 214-221.

Huff-Lonergan, E., \& Lonergan, S. M. (2005). Mechanisms of waterholding capacity of meat: the role of postmortem biochemical and structural changes. Meat Science, 71, 194-204.

Leksrisompong, P. N., Lanier, T. C., \& Allen Foegeding, E. (2012). Effects of heating rate and $\mathrm{pH}$ on fracture and water-holding properties of globular protein gels as explained by micro-phase separation. Journal of Food Science, 77(2).
Lin, Z., Chen, H., \& Lin, F. (2001). Influence of pretreatments and blanching treatments on the yield and color of canned mushrooms. Journal of Food Processing and Preservation, 25(6), 381-388.

McArdle, F. J., Kuhn, G. D., \& Beelman, R. B. (1974). Influence of vacuum soaking on yield and quality of canned mushrooms. Journal of Food Science, 39(5), 1026-1028.

Nicolai, T., \& Durand, D. (2013). Controlled food protein aggregation for new functionality. Current Opinion in Colloid and Interface Science, 18(4), 249-256.

Paudel, E., Boom, R. M., \& van der Sman, R. G. M. (2015). Change in water-holding capacity in mushroom with temperature analyzed by Flory-Rehner theory. Food and Bioprocess Technology, 8(5), 960970.

Pearce, K. L., Rosenvold, K., Andersen, H. J., \& Hopkins, D. L. (2011). Water distribution and mobility in meat during the conversion of muscle to meat and ageing and the impacts on fresh meat quality attributes - a review. Meat Science, 89(2), 111-124.

Schijvens, E. P. H. M., Ruisch, H., van Deelen, W., Biekman, E. S. A., \& Wichers, H. J. (1996). The density of common mushroom (Agaricus bisporus) tissue related to the shrinkage at heating. Mushroom News, 44(9), 14-19.

Shahraeeni, E., \& Or, D. (2010). Pore-scale analysis of evaporation and condensation dynamics in porous media. Langmuir, 26(5), 13924 13936.

Tuller, M., Dani, O., \& Dudley, L. M. (1999). Adsorption and capillary condensation in porous media: liquid retention and interfacial configurations in angular pores. Water Resources Research, 35(7), 1949-1964.

Urbonaite, V., De Jongh, H. H. J., Van Der Linden, E., \& Pouvreau, L. (2015). Water holding of soy protein gels is set by coarseness, modulated by calcium binding, rather than gel stiffness. Food Hydrocolloids, 46, 103-111.

Van der Sman, R. G. M. (2008). Prediction of enthalpy and thermal conductivity of frozen meat and fish products from composition data. Journal of Food Engineering, 84(3), 400-412.

Van der Sman, R. G. M. (2015). Biopolymer gel swelling analysed with scaling laws and Flory-Rehner theory. Food Hydrocolloids. doi:10. 1016/j.quageo.2014.03.002.

Van der Sman, R. G. M., Ekaraj, P., Voda, A., Khalloufi, S., \& Khallou, S. (2013). Hydration properties of vegetable foods explained by FloryRehner theory. Food Research International, 54(1), 804-811.

Zivanovic, S., Busher, R. W., \& Kim, K. S. (2000). Textural changes in mushrooms (Agaricus bisporus) associated with tissue ultrastructure and composition. Journal of Food Science, 65(8), 1404-1408.

Zivanovic, S., Buescher, R., \& Kim, S. K. (2003). Mushroom texture, cell wall composition, color, and ultrastructure as affected by $\mathrm{pH}$ and temperature. Journal of Food Science, 68(5), 1860-1865. 